\title{
A Formal Approach for the Inclusion of Key Performance Indicators in a Business Process Modeling
}

\author{
Atsa Etoundi Roger \\ Department of Computer Sciences, \\ University of Yaoundé I, \\ Yaoundé, Cameroon
}

\author{
Fouda Ndjodo Marcel \\ Department of Computer Sciences, \\ University of Yaoundé I, \\ Yaoundé, Cameroon
}

\author{
Atouba Christian Lopez \\ Department of Computer Sciences, \\ University of Yaoundé I, \\ Yaoundé, Cameroon
}

\begin{abstract}
Modeling business processes is very complex because it requires bringing together several dynamic parameters. Notably, quality requirements of customers of the organization, business process related tasks and requirements expressed by business executives of the organization each being considered by its level of importance. In this paper, we propose an approach based on two axes which aim at merging three dynamic parameters in modeling a business process. The first theme revolves around two main activities: (i) identification of viewpoints of the beneficiaries of business process services; (ii) the definition of indicators and performance factors of the organization as well as quality factors of clients of the said organization. In the second axis, we shall demonstrate, (i) the validation of levels of importance associated with the expressed requirements by business executives; (ii) the impact of this approach in the definition of business process activities (iii) the critical deduction scheme and the critical business processes requirement model, depending on the quality factors of the organization's customers. The diagram of a critical process is the set of tasks essential to the satisfaction of customers of that organization. Quality factors are subjective information from which the beneficiary rests his judgment on the service rendered by the business process. It was noted at the end of this paper, a new vision towards which modeling business processes seeks to arrive at. This vision was named the modeling approach by triangulation of business processes.
\end{abstract}

\section{General Terms}

Key Performance Indicator.

\section{Keywords}

Business Process Modeling, Requirement Engineering, Software Component, Specification language, Application Engineering, Requirement representation, Quality of Service, Business Process Quality of Service, Key performance Indicator.

\section{INTRODUCTION}

In [1], it was proposed a goal-oriented approach for developing a business process requirement model. This approach has at its core, a formal representative structure of the requirements of the organization. This formal structure uses amongst others the level of importance of user needs. However, no guideline was given as to how to validate it, neither on how to assign a value to the latter. This concept of "importance level" in the context of business process modeling has been widely reported in the literature. In $[1,2,3$, 4], it allows the definition of a priority order between concepts of the same nature ("Business rules", "expressed requirement", "user requirements ", "tasks", etc..) but none of these authors does address the concerns mentioned above. It appears, however, from their work that the level of importance affects the "quality service" of a computer application. We believe, therefore, that these studies all contribute to achieving a single objective: mastery of user requirements in order to minimize misunderstandings discussed in $[6,7,8]$, between computer application users and designers of such applications and, incidentally, to anticipate their evolution thereby improving the life cycle of applications resulting from these requirements. Despite the relevance of their work, it seemed that the authors were not much interested, in literature, in the formal conciliation of the internal quality of service within business processes, the level of importance associated to user expectations, and quality of service offered to clients of the organization (service offered). Part of this problem has been addressed in the thesis of (Anis Ferchichi, 2008). But it was limited to developing a model to incorporate the recommendations of the qualities of different standards (ISO 9001v2000 and CMMI) to create a single repository. However, we believe that, as needs change, expectations of the beneficiaries of services offered by business process change as well and it is not sufficient to be limited to (ISO and CMMI 9001v2000) standards, but instead to integrate quality of service provided as quality requirements or quality demands.

In this context, it becomes essential to have: (1) a formal approach for evaluating the quality of service provided to enable the organization to fine-tune based on the quality requirements of its clients, activities of its business processes with a view to maintaining or improving the quality of service provided to such clients. This involves the need for performance indicators for each task of the organization's business processes, (2) means of obtaining quality demands of service consumers in order to anticipate their evolution and take this into account in modeling business processes, and in addition to user requirements and their level of importance, client satisfaction. Which will enable us for sure to: - develop computer applications with a known degree of quality from the analysis phase, - develop services tailored to each client of the organization, dynamically-evaluate the quality of service of a business process , - identifying unnecessary levels of importance in the organization's requirements, - generate a critical chart for business processes according to the target quality level, - identify unnecessary tasks in business processes, based on the quality target. We believe a solution can be found to the previous wishes.

We propose an approach based on two axes which aim at merging three dynamic parameters in modeling a business process. The first point revolves around two main activities: (i) identification of viewpoints of the beneficiaries of business process services; (ii) the definition of indicators and performance factors of the organization as well as quality factors of clients of the said organization. In the second point, we shall demonstrate, (i) the validation of levels of importance associated with the expressed requirements by business executives; (ii) the impact of this approach in the definition of business process activities (iii) the critical deduction scheme and the critical business processes requirement model, depending on the quality factors of the organization's 
customers. The diagram of a critical process is the set of tasks essential to the satisfaction of customers of that organization.

In continuance, our work will be organized as follows: in Section two (2), we shall present, in a general manner business processes, in Section three (3), we shall discuss the basic concepts, and Section four (4) is dedicated to , identifying quality expectations, while Section five (5) will be dedicated to the definition of performance indicators for each task of a business process; Section six (6) shall discuss, the definition of the organization's quality function, and we complete with Section seven (7), which is dedicated to the conclusion and future works.

\section{BUSINESS PROCESSES}

Usually, a business activity is considered a hierarchical organization that reflects the functional decomposition of the enterprise and it's command chain. The various departments are specialized in specific functions (eg sales, production or accounting), and in each department, sub-departments, teams or individuals specialize in sub-functions. For example, the processing of an order from a customer crosses different departments: sales (to receive the order), planning (to plan the manufacture of the product or the completion of the inventory), production and dispatch and accounting. Early theories of business management focused on business operations and efficient control of its departments (command chain, workflow, communication, etc.) by focusing on each separate department. With the advent of BPM (Business Process Management) and BPR (Business Process Reengineering), a revolution took place: instead of focusing on each function of the enterprise separately, and therefore without questioning overall structure of the process, researchers have suggested that we look at the entire business process from beginning to end, while seeking to optimize the entire processes. Business processes are, therefore, the representative processes of the activities of the enterprise independently of human and technical resources. Each business process is identified by at least an objective and its degree of success qualitatively or quantitatively measurable. The activities of a business process are performed by actors playing particular roles, consuming resources and producing others. The researchers recommend that these activities are called tasks $[9,10]$. They proposed to this effect, in [28] that a business process is represented formally by:

\section{$\langle$ Observ,Tasks, follow, goal $\rangle$}

where:

-Observ is the set of observers. An observer is a Boolean variable that describes a reality.

- Tasks is the set of tasks

- follow : Tasks $\rightarrow 2^{\text {Tasks }}$ is a function that gives for each task the set tasks which are next to it if the conditions are fulfilled

-goal is the observation that one seeks satisfaction after the process. An observation is, what the user sees at the end of the execution of a task.

While a task itself will be formally represented by:

$\langle$ Name_of_task,precondition,postcondition $\rangle$

- Name_of_task is the name of the task
- precondition is an observation that must be satisfied before

- postconditon is an observation that is guaranteed after the execution of the task

However, this modeling of business processes ignores the requirements inherent to each business process and the importance of certain activities relative to others. In the literature, a number of authors have focused their research on the modeling requirements of business processes. What emerges from this research is reported in $[14,1,2]$. The following paragraph is a summary of the work of $[14,1,2]$.

\subsection{Needs of a Business Process}

In the literature, several authors have made attempts on the problem of business process requirement modeling, but some such as $[14,1,2]$ addressed the problem from a formal angle. Frida Semmak and Joël Brunet in 2005, proposed in [14], a goal oriented meta-model for specification of the requirements of a domain. Their work was completed in 2010 , [1,2] with the introduction of new concepts in the formal representation of a requirements proposed in [14]. The approach proposed in [1] extends the representation of requirements of Joël Brunet and frida Semmak so that it can integrate concepts of importance of level, domain, constraints. The approach proposed by [1] is goal oriented for the definition of a business process requirement model, taking into account their level of importance and constraints inherent to these requirements. The level of importance of a goal is the credit that a user gives to this goal. Constraints are non-functional requirements relative to the goal that must be met. The representation of the expressed requirements or knowledge bits proposed in [1] is defined as follows:

$$
\begin{aligned}
& \text { Where: } \quad \partial=(\psi, \omega, \lambda, \delta, v) \\
& \partial \text { is the name of knowledge bit } \\
& \psi \text { is the context in which the goal is set } \\
& \omega \text { is the goal } \\
& \lambda \text { is the business rule } \\
& \delta \text { represents the contraints } \\
& v \text { is the level of importance of the goal } \\
& \partial \text { represents name of a domain concept } \psi \text {. }
\end{aligned}
$$

This approach revolves around four main principles: elicitation of user requirements, selection of different goals, and transformation of necessities to bits of knowledge and finally the development of the requirements model. This approach has as advantages: - reducing misunderstandings raised by [6] [7] [8] between application developers and users; - the understanding of user requirements in the formal representation of such requirements; and finally, the integration of the level of importance of various aspects of the system and the constraints inherent to these requirements. However, this approach has as its main weakness: the lack of guidelines on the validation of the level of importance attributed to requirements.

\subsection{Quality of Service of a Business Process}

The quality of Service represents an abstract concept, disperse and multifaceted. Originally, the foundations of the theory of quality of service are based on the concepts of product quality and consumer satisfaction. Early conceptualizations (eg Grönroos in [16, 17] Parasuraman, Zeithaml and Berry [19]) 
were based on the disconfirmation paradigm used in the literature on physical products (eg, Cardozo in [15] ,Churchill and Surprenant, in [25] Howard and Sheth in [22] Oliver in [20]; Olshavsky and Miller in [24]) This paradigm states that the quality results of a comparison between what is perceived and the expected performance. Unlike the quality of goods that can be objectively measured by indicators such as durability or the number of manufacturing defects, quality of service is a abstract constructed and diffused because of three characteristics related to services: intangibility, heterogeneity and inseparability (Eiglier Langeard and in [23]). The research in this area focuses on the quality perceived by the consumer. In 1988, Parasuraman, Zeithaml and Berry in [18] have made an important contribution in this research area by developing an instrument for measuring the perceived quality of a service.

In effect, whether objective quality is high or not, the impact of quality of service is realized via that perceived by the consumer. It, in fact represents, the consumers judgment on the degree of excellence or superiority attributed to an entity (Parasuraman et al. In [21]). Moreover, the quality is never acquired, it is always relative: this relativity is expressed for a single person obtaining the same service, but in different situations. Moreover, the concept of quality of service is doubled, it represents both a state and a process. Parasuraman, Zeithaml and Berry in [18] suggest that the quality of service is the result of the difference between consumer expectations (ie what he considers to be the service offered by the organization) and the evaluation of the actual performance of the service. The Perceived quality of service is conceptualized and operationalized as the difference or gap between expectations and perceptions, difference from the perspective of its amplitude and direction. (Anis Ferchichi, 2008) in [5] notes two types of qualities coexist, internal quality of service (process quality) and external quality of service (quality of result). External quality, corresponding to customer satisfaction. This is about providing a product or services in conformity with the expectations of clients in order to retain and improve market share. The beneficiaries of the external quality are the clients of a company and its external partners. This type of approach necessarily requires listening to clients but must also allow to take into account the implicit requirements, not expressed by consumers; the internal quality, corresponding to the improvement the internal functioning of the company. The purpose of internal quality is to implement ways to best describe the organization, identify and mitigate failures. The beneficiaries of the internal quality are the management and staff of the company. Internal quality generally goes through an identification and formalization stage of internal processes achieved through a participatory approach. The purpose of quality of service is to provide targeted offers to clients, with controlled processes, while ensuring that the improvement does not result into an increase in the product price.

The simultaneous nature of the perceived quality of service and the process seems to have led researchers to be interested to reconcile the two aspects of quality of service (internal QoS, external QoS). We believe that this model is possible since, in as much as when modeling a business process, there is at least an organization that makes use of the business process. Therefore, clients of this organization can be found. Thus, it becomes easy to collect their perceptions of quality of service in this organization and make projections on the quality of service for the future system. In this perspective, Carman [29] proposes to know the weight of each attribute in evaluating the quality of service in order to distinguish determining attributes from important and especially conspicuous determinants. A preliminary qualitative study appears to be the best way to know the importance of the attributes of a service.

In the literature, the authors do not seem interested in the importance of certain activities relative to others, and do not reconcile the quality of services perceived by consumers of the services of a business process to performances of activities of this business process. We believe that reconciliation is possible. In the next section we present an approach for the above mentioned reconciling in modeling a business process.

\section{BASIC CONCEPTS}

In the previous section, we presented, in a general manner, the different facets of a business processes. A number of weaknesses were also noted on each of the above mentioned facets. In this section, we shall firstly, formalize the different basic concepts; secondly we shall present the impact of quality of service on the latter.

\section{Definition (1): Observer}

An observer is a variable attached to a business rule or a business object of a business process, whose content is updated during the execution of an activity of this business process making use of the said business rule, or business object.

\section{Definition (2): Observation}

An observation is a Boolean variable.

We shall consider, Observers as any observers of a business process and $\operatorname{card}(\mathrm{X})$ represents the cardinality of a set X.

\section{Definition (3): Evaluation Function}

Consider a function $\mathrm{f}_{\text {eval }}$ : Observers $\rightarrow[0,1]$, that for all Obs element of Observers, $\left.f_{\text {eval }}(\mathrm{Obs})\right)$ returns a numeric value representing the content evaluation of the observer Obs. $f_{\text {eval }}$ is called evaluation function.

\section{Definition (4): Observation Function}

Consider a function $f_{t h}: R \rightarrow\{0,1\}$ (where $t h$ is a numerical value representing the threshold), which for any real $a$,

$$
f_{t h}(a)=\left\{\begin{array}{l}
0, \text { if } a<t h \\
1, \text { else }
\end{array}\right.
$$

\section{Definition (5): Indicator}

An indicator is information that helps in the appreciation of a situation. It represents the result of the evaluation of an observer associated with a business process. Formally, an indicator associated with an observer $b(b \in$ Observers $)$ shall be defined as follows: $e=f_{\text {eval }}(b)$.

We shall consider the following IndiQ as the set of indicators of a business processes. It is necessary to note that $f_{\text {eval }}$ is a family of functions.

\section{Definition (6): Observation of an Indicator}

Consider an indicator $e \in$ IndiQ and $b \in$ Observers, the observer associated to $e$ (with $e=f_{\text {eval }}(b)$ ), we define an observation of $e$ as a threshold function $g_{t h}$ : IndiQ $\rightarrow\{0,1\}$ such that :

$$
g_{t h}(e)=\left\{\begin{array}{l}
0, \text { if } f_{\text {eval }}(b)<t h \text { where: } \text { th is the } \\
1, \text { else }
\end{array}\right.
$$
satisfaction threshold $(t h \in] 0,1[$, 
$\underline{N B}$ (1): Without distorting the generality, $\left.f_{\text {eval }}(b)\right)$ may from time to time be replaced by a real value in the interval $[0,1]$.

\subsection{Viewpoint}

The point of view of a client relative to a service that is rendered to him in an organization represents the feeling relative to the service that was rendered to him in this organization. According to [29], this point of view is based on a set of observations of which the importance is worth knowing. The relevance of modeling views of customers of an organization are justified insofar as the client of an organization is the main consumer of the service rendered by this organization. And, not taking into account the opinion of the latter may lead to the organization losing customers in favor of competing organizations. The complexity in taking into account the views of customers of an organization comes from the fact that: the point of view is relative and depends on each client, point of view of a customer follows the service that the client received. We believe, however, that if the business process is customer satisfaction oriented, it is possible that the latter is adjusted over time according to the guidelines of the client. Two concepts are important for the representation of view point of clients: the quality factors;knowledge of customer satisfaction.

\subsubsection{Quality Factor}

A quality factor is an observation from which a customer uses to give his judgment relative to the service that was rendered. Quality factors are intimately linked to the service enjoyed by the customer. Basing on the work of [29], we define a quality factor fact as follows:

$$
\text { fact }=\langle\text { value, } \text { Poids }\rangle
$$

where :

$$
\begin{aligned}
& \text { - value is an observation ( value } \in\{0,1\} \text { ); } \\
& \text { - Poids: represents the importance of the } \\
& \text { observation value in the satisfaction of the } \\
& \text { customers. }
\end{aligned}
$$

We shall henceforth consider QualityFactors as the set of quality factors on which is based the judgments of the service consumers of a business processes. We denote $\mathcal{P}$ (QualityFactors) is the set of parts of QualityFactors

\section{Definition (7): Satisfaction Indicator}

Consider a client $c l$ and a service $S$ he received in an organization. Suppose that $W$ is the set of quality factors based on which $c l$, judgement is based on, we define a function $h:($ QualityFactors $) \rightarrow[0,1]]$ such that $W$ element of $\mathcal{P}$ (QualityFactors),

$$
h(W)=\frac{\sum_{\text {fact }=\langle\text { val }, p\rangle \in \text { QualityFactors }}(\text { fact } . p \times \text { fact. val })}{\sum_{\text {fact }=\langle\text { val }, p\rangle \in \text { QualityFactors }} \text { fact. } p}
$$

$h(W)$ is called an indicator of customer satisfaction

\section{Definition (8): Job Satisfaction}

Consider a client $\mathrm{cl}$ and a service $S$ he received in an organization. Suppose that $W$ is the set of quality factors based on which $\mathrm{Cl}$ judgment is based, we define a threshold functionSat $_{t h}: \mathcal{P}$ (QualityFactors $) \rightarrow\{0,1\}$ such that, $W$ element of $\mathcal{P}$ (QualityFactors),

$$
\text { Sat }_{t h}=\left\{\begin{array}{l}
0 \text { if } h(w)<t h \\
1 \text { else }
\end{array},\right.
$$

th $\in[0,1]$ represents the satisfaction threshold of customer $c l$

$N B$ (2): without deviating from the generality, we shall replace $h(w)$ by its numerical value.

A point of view $\mathrm{Pv}$ of a client being the judgment that a client associates to a service that is rendered to him in an organization, we represent it formally as follows:

$$
\mathrm{Pv}_{\mathrm{cl}}=\langle\mathrm{D}, \mathrm{cl}, \mathrm{S}, \mathrm{W}\rangle
$$

Where :- $\mathrm{cl}$ : is the name of a user;

$-\mathrm{S}$ is the service that has benefited;

$-\mathrm{W}$ : is the set of observations of client cl;

-D : is the context of service

-S Knowledge of Satisfaction

\subsubsection{Knowledge of Customer Satisfaction}

The knowledge of customer satisfaction represents the set of information of the business process required to calculate the quality factors. We define formal manner knowledge of satisfaction $C s$ as follows:

$$
\mathrm{Cs}=\left\langle\mathrm{D}, \mathrm{S}, \mathrm{V}_{\mathrm{W}}, \Delta_{\mathrm{cl}}\right\rangle \text { where: }
$$

-D : is the knowledge domain;

$-\mathrm{S}$ : represents the service under evaluation;

$-V_{W}$ : represents the set of observers whose evaluation led to the set of observations of $W$;

$-\Delta_{c l}$ : represents the threshold of customer satisfaction of the $c l$

\subsection{Constructing the Set of Viewpoints}

\subsubsection{Identification of viewpoints}

This activity aims at obtaining the set of viewpoints of customers of the organization. It is continuous and allows an organization to focus its management on customer satisfaction. The viewpoints collected must respect the previous structure. We denote by $P_{\text {views }}$, the set of viewpoints of customers of the organization.

3.2.2. Rules for construction of all quality factors Once collected the different viewpoints of customers of the organization, we will build a MultiSet, $m_{-}$Observations and Observations the set of observations of the various beneficiaries of the service rendered by a business process, as follows:

$$
\begin{gathered}
m_{-} \text {Observations }=\coprod_{p v=(d, c l, s, w) \in P_{\text {views }}} p v \cdot w \\
\text { Observations }=\bigsqcup_{p v=(d, c l, s, w) \in P_{\text {views }}} p v \cdot w
\end{gathered}
$$

Where: $-\amalg_{*} *$ : represents a union of set with repetition of elements. This operator allows the construction of a mulitset.

- $U_{*} *$ : represents the union set operator. 
m_Observations: is a collection of observations of each beneficiary of a service provided by the business process, Observations is a set of observations of different beneficiary of this service. We define the function $f_{o c c}: m_{-}$Observations $\rightarrow N$ such that whatever obs $\in$ m_Observations, $\mathrm{f}_{\text {occ }}$ (obs) returns the number of occurrences in m_Observations. We shall construct the set of quality factors (QualityFactors) as follows:

$$
\text { QualityFactors }=\bigcup_{o b s \in m_{-} \text {observations }}\left(o b s, f_{o c c}(o b s)\right)
$$

$f_{o c c}(o b s)$ shall represent the weight of the observation obs.

\section{Axiom 1: Divergent Viewpoints}

Consider two viewpoints $P v_{1}$ and $P v_{2}, P v_{1}=(d, c l, s, w) \in$ $P_{\text {views }}$ and $P v_{2}=(a, c u s t, b, v) \in P_{\text {views }}, P v_{1}$ and $P v_{2}$ shall be said divergent if and only if $P v_{1} \cdot w \cap P v_{2} \cdot v=\emptyset$.

\section{Axiom 2: Similar Viewpoints}

Consider two $P v_{1}$ and $P v_{2}, P v_{1}=(d, c l, s, w) \in P_{\text {views }}$ and $P v_{2}=(a$, cust $, b, v) \in P_{\text {views }}, P v_{1}$ and $P v_{2}$ Shall be said to be similar if and only if $P v_{1} . w \cap P v_{2} . v=P v_{1} . w$ or $P v_{1} . w \cap$ $P v_{2} \cdot v=P v_{2} \cdot v$.

\section{Necessary conditions 1: Convergence Criteria}

Consider two partitions of $A_{\text {fact }}$ and $B_{\text {fact }}$ of QualioyFactors, $A_{\text {fact }}$ is the set of quality factors for which the weight is equal to $1 ; B_{\text {fact }}$ is the set of quality factors for which the weight is different from 1 . We say that all quality factors QualityFactors are convergent if and only if $\operatorname{card}\left(B_{\text {fact }}\right)>\operatorname{card}\left(A_{\text {fact }}\right)$. QualityFactors is divergent otherwise.

\section{KEY PERFORMANCE INDICATORS}

\subsection{Performance Observers}

A performance observer is a set of information associated to a task or a business object from which we can measure the performance of a task or the state of progression or processing of a business object in a business process. The modeling of a performance observer $O p$ associated with a task $t$ is defined as follows:

$$
O p=\left\langle C, t, \Sigma_{o b s}\right\rangle
$$

Where: $-t$ is a task or a business object;

$$
\begin{aligned}
& -\Sigma_{o b s} \text { represents all observers associated with } \mathrm{t} \text {; } \\
& -C \text { : represents a context or domain. }
\end{aligned}
$$

Performance observers are updated by the execution of activities to which they are attached. In the following, PerfObservators denote the set of observers performance and $p O b s(t)$, the set of performance observers associated to $t$.

\subsection{Performance Indicator}

Consider an activity $t$ of a business processes; $O p \in$ PerfObservators the performance observer associated with the activity $t ; \mathcal{P}$ (PerfObservators) the set of parts of Perfobservators; we define the function: $f_{\text {Ind }}: \mathcal{P}($ PerfObservators $) \rightarrow[0,1]$ which is such that whatever an performance observer $O p$,

$$
f_{\text {Ind }}\left(O p \cdot \Sigma_{o b s}\right)=\sum_{o b s \in O p \cdot \Sigma_{o b s}} \frac{f_{\text {eval }}(o b s)}{\operatorname{card}\left(O p \cdot \Sigma_{\text {obs }}\right)}
$$

The function $f_{\text {Ind }}$ is called the performance indicator of the activity $t$. No restrictions are made on the number of performance observers associated to an activity. It therefore may occur that a task may have several performance indicators. It will be the same for a business object. We denote IndiQ $(t)$ the set of indicators associated to $t$ where $t$ can be either a business object or a task.

Performance indicators are interested by internal quality of service of the business processes of an organization. It is noted in [30], the existence of several categories of indicators:

- Indicators alert: This type of indicator 'all or nothing type signals an abnormal state of the system under control requiring action immediately or not. an critical threshold crossing for example falls into this category of indicator;

- Indicators balancing: This type of indicator, closely linked to the objectives, is a little compass of the decision maker. He informs on the state of the system under control in relation with the objectives pursued. Will they be kept?

- Indicators of anticipation: A good dashboard is also an instrument of foresight. A good dashboard lets see a little further than the tip of the screen and consider with a better foundation for the current situation. Should we continue with the current plan? Revise?

It happens very often that performance indicators are dependent on each other. In the Directorate of Career Management in the Ministry of Public Service and Administrative Reform in Cameroon, the indicators used are dashboards. It is found that the dashboard of the Assistant Director of Personal officials depends largely on those services which are attached; and dashboards of these heads of each service depend in large part to those offices which are attached. All these dashboards are generated manually and opening the door for errors.

\section{Axiom 3: Constraints Defining Indicators}

(1) The performance indicators are defined by the top-down approach (from the apex of the tasks to tasks located at the last level of the hierarchy).

(2) The performance indicators of the top of the hierarchy of tasks must be defined in relation to the observations of the beneficiary of service provided by the business process.

Axiom 4: Dependence between Performance Indicators Let's consider $t$ a task; $\operatorname{task}(t)$ a set of sub-tasks of the task $t$; typeof (Ind) (with Ind $\in \operatorname{IndiQ}(t)$ ) the type of indicator Ind; and $\operatorname{Indi} Q_{s}(t)$ the set of indicators of $s$ type associated with sub-tasks of $t$,

(3) $\operatorname{task}(t)=\phi \quad, \quad \forall$ Ind $\in \operatorname{IndiQ}(t), \exists o=\left(t, c, \Sigma_{\text {obs }}\right) \in$ $p O b s(t) /$ Ind $=f_{\text {Ind }}\left(o . \Sigma_{\text {obs }}\right)$.

(4) $\operatorname{task}(t) \neq \phi$

(a) $\forall t^{\prime} \in \operatorname{task}(t), \operatorname{Indi} Q\left(t^{\prime}\right) \cap \operatorname{Indi} Q(t)=\operatorname{Indi} Q\left(t^{\prime}\right)$

(b) $\forall$ Ind $\in \operatorname{IndiQ}(t), \exists ! \operatorname{Ind}^{\prime} \in \operatorname{IndiQ}\left(t^{\prime}\right) /$ typeof $($ Ind $)=$ typeof $\left(\right.$ Ind $\left.^{\prime}\right)$

(c) the value of Ind indicator Ind $\in \operatorname{IndiQ}(t)$ depends of those of the performance indicators of its sub-tasks.

Definition (9): Performance Factors

A performance factor is a triplet $\langle$ Indicator, $w, t\rangle$ where $t$ is a task; Indicator $\in \operatorname{IndiQ}(t)$ and $w$ the weight of Indicator indicator.

Axiom 5: Dependence between the Performance Factors 
(5) Two performance factors factPer $_{1}=\left\langle\right.$ Ind $\left._{1}, w_{1}, t_{1}\right\rangle$ and factPer $_{2}=\left\langle\operatorname{Ind}_{2}, w_{2}, t_{2}\right\rangle$ are called dependent if and only if $\operatorname{Indi} Q\left(t_{2}\right)$ and $\operatorname{Indi} Q\left(t_{1}\right)$ are dependent;

(6) Consider a performance factor facper $=$ $\langle$ Ind,weight, $t\rangle$, we assume $\operatorname{task}(t) \neq \phi$. We shall also consider that factors $(t)$ the set of performance factors associated to $t$; and $\operatorname{subfactor}_{s}(t)$ the set of performance factors associated with the sub tasks $t$ for which performance indicators are of type $s$.

(a). The value facper. Ind is calculated as follows:

$$
\text { facper.Ind }=\frac{\sum_{I d=\langle i, w, s\rangle \in \text { subfactors }(t)} i d . i \times i d . w}{\sum_{o=\langle j, v, l\rangle \in \text { subfactors }_{s}(t)} \text { o.v }}
$$

(b). The value facper. $w$ shall be determined as follows:

$$
\text { facper.weight }=\max \left(\bigcup_{o=\langle i, w, s\rangle \in \text { subfactors }_{S}(t)}\{o . w\}\right)
$$

Where: $s=$ typeof (facper. Ind $)$ and $\max$ returns the largest element in the list of items received as a parameter.

\section{Definition (10): Performance of a Business Process}

We consider $t$, a task of a business processes as being the root of the hierarchy of tasks of business processes. We define the function power $_{\text {del }}: \mathcal{P}$ (PerfObservators $) \rightarrow[0,1]$ such that for every element $w$ of $\mathcal{P}$ (Perfobservators),

$$
\operatorname{power}_{\text {del }}(w)=\frac{\sum_{a=(i, p) \in w} f_{d e l}(a \cdot i) \times a \cdot p}{\sum_{b=(j, p) \in w} b \cdot p}
$$

\section{APPROACH FOR THE INTRODUCTION OF PERFORMANCE INDICATORS}

In the previous sections, we presented the basic concepts necessary for understanding our approach. In this section we present our approach.

\subsection{Approach}

This approach aims to introduce performance indicators in business processes. However, the introduction of indicators focuses on customer satisfaction. Our approach is structured around four main points: (i) construction of the set of clients observations of the organization, (ii) construction of quality factors, (iii) construction of the sets of observers associated with each observation, (iv) construction of performance factors. The points (i) (respectively (ii)) have been sufficiently detailed in Section 3.2.1 (respectively 3.2.2). As a result, they are no longer addressed.

\subsubsection{Construction of Observers}

The construction of observers is equivalent to defining the set of variables from which the indicators shall be calculated. Consider a quality factor fact element of QualityFactor, the construction of the set of observers is manual and dependent on the analyst and requires compliance with the following steps:

(1) define from the bottom to top, observers from which performance indicators of each task shall be calculated. All the tasks of a business processes must be taken into account;

(2) define evaluation functions for each observer $f_{\text {eval }}$ an elementary task (tasks found at the last level of the tasks hierarchy);
(3) define the threshold satisfaction associated with;

This process must be repeated for the set of quality factors constructed in Section 3.2.2. This process helps to construct the function $h_{\text {map }}:$ QualityFactors $\rightarrow \mathcal{P}$ (Observators) that for any quality factor fact $\in$ QualityFactors, returns the set of observers associated with the quality factor fact; and the function $h_{\text {task }}:$ Observators $\rightarrow$ Tasks that for any observer who , returns to the task to which the observer is associated with. We shall note Observator $_{Q f}(t)$, the observer associated with the task $t$ to the achievement of the quality factor $Q f$.

\subsubsection{Construction of Performance Factors}

The construction of performance factors should be done by software analysts assisted by business executives of the organization. This construction is based on the set of observers defined below to which is added the weight of each indicator. In this section the indicators are calculated values, they shall therefore be deduced from the results of Section 5.1.1. Construction performance factors therefore require the definition of the weights associated with each indicator. Thus, given a quality factor fact $=\langle$ value, weight $\rangle \in$ QualityFactors, elemTasks the set of elementary tasks of a business process and a task $t \in$ elemTasks; the definition of the weights associated with performance indicators imposes respect of the steps below:

(1) if $t$ helps to achieve the observation fact.value then the performance factor $\mathrm{fp} f p=\langle$ ind, $w$, task $\rangle$ associated with $t$ for satisfy the quality factor fact is defined as follows: $n d=f_{\text {eval }}($ Observator $(t)) ; w=$ fact. weight $; t i=t$;

(2) if $t$ does not contribute to the achievement of the result,: ind $=f_{\text {eval }}($ Observator $(t)) ; w=0 ; t i=t$.

(3) for intermediate tasks and root task of the tasks hierarchy of business processes, performance factors will be determined according to the axiom 5 - (2).

\subsection{Impact on the Requirements Model}

The impact of the introduction of performance indicators in the modeling of business processes is more felt in the validation levels of importance associated with each expressed requirement by business executives of the organization. Thus, given a task $t$ a quality factor $f q$, an expressed requirement $b$. We assume that $b$ is associated with the task $t$. Consider a performance factor $f p$ also associated to $t$. We define a majority function maj:Tasks $\rightarrow$ boolean which is such that for whatever task $t \operatorname{maj}(t)$ returns 'true' if the number of performance factors associated with the task $t$ with a nonzero weight is greater than those whose weight zero, and 'false' otherwise.

\section{Axiom 6: Important requirement relative to a Threshold}

Consider a requirement $\mathrm{b}$ and a threshold del, we say that the requirement $b$ is important if its level of importance is above the threshold del. del is called threshold of importance of the expressed requirement.

\section{Axiom 7: Consistency between Requirements and} Performance Indicators

Consider a need expressed $\mathrm{b}, \mathrm{t}$ associated with a task and $\mathrm{b}$ need a materiality threshold del, we say that the level of importance nor $b$ is an expressed need

(7) Coherent with performance indicators if and only if $\operatorname{maj}(t)$ has the value 'true' and $n i>$ del. 
(8) as otherwise incoherent;

(9) Performance factors associated with t are also known as important factors associated with the expressed requirement $b$. It is the same for the other concepts mentioned above.

In case of incoherence between the level of importance of a requirement and performance indicators associated with this requirement, the software analyst should refer to the business executives to adjust the level of importance of the expressed requirement.

\section{Definition (11): Critical Requirement}

(1) Consider an expressed requirement $b$ and a task $t$ associated with the expressed requirement $b$, we shall say that $b$ is a critical requirement if and only if its level of importance is consistent with the performance indicators associated with $\mathrm{t}$.

(2) the set of critical requirements form the critical business process requirement model.

In the work of [31], it was defined an approach for specifying the business rules of a business process, due to what preceded, it is necessary that the business rules support the concepts of quality observers. For this, the following constraint has been issued.

\section{Constraint: Business Rule}

(1) Observers must be declared in the context of each business rule through the type Observer. They represent a pair (variable, weight);

(2) observers declared in the context of a rule must be updated during the execution of the rule;

(3) an indicators evaluation rule must be defined.

\subsection{Impact on the Definition of Tasks}

\section{Definition (12): Critical Tasks}

(1) A task $t$ of business processes shall be called critical task if and only if maj(t)is 'true'.

(2) The set of critical tasks of a business process form the critical chart of the latter.

In the work of [28], a definition of a task was proposed in the line with the previous sections, we propose to take into account the performance factors in the definition of a task. Thus, the new definition of a task $t$ in business processes is presented as follows:

$\mathrm{t}=\langle$ Name_of_task, precd, postcd, perfobservators $(\mathrm{t})\rangle$ where :

- Name_of_task is the name of the task

- precd is an observation that must be satisfied before

- postcd is an observation that is guaranteed after the execution of the task

- $\operatorname{perFactors}(t)$ is the set of observers associated with the performance task $t$.

\subsection{Impact on Business Process Modeling}

From above, we believe that the modeling of business processes must respect the axes in the diagram below.

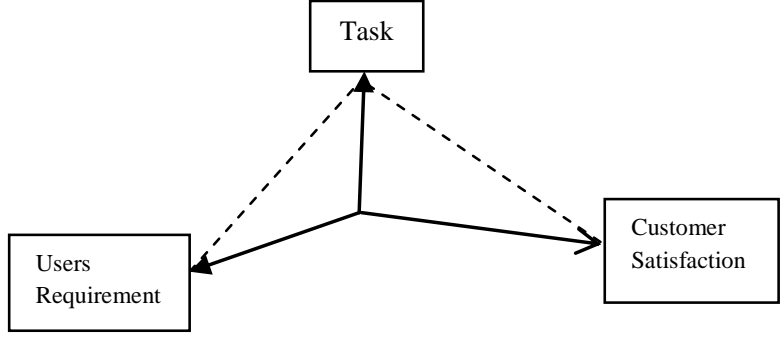

Figure 1: Business Process Modeling Model

This chart recommends that when modeling a business process, the software architect should take into account user requirements, tasks of business processes and the satisfaction of consumers of the services rendered by the business process. We call this approach in the business processes modeling, Triangular Modeling Approach (TMA) or Star Modeling Approach (SMA). This approach has a huge advantage over development procedures based on domain engineering since there is consideration of the satisfaction of consumers of business processes, which is what domain engineering approaches do not do.

\section{CONCLUSION AND FUTURE WORKS}

We presented in this paper, an approach for the introduction of performance indicators in the modeling of business processes. This introduction of performance indicators requires consideration of satisfaction of the consumers of the service rendered by the business process. This on the one hand, has induced a set of impact on the requirement model and in particular on business rules, on the definition of a business task and on the other hand, has permitted us to define the concept of coherence between levels of importance of a requirement and performance indicators, a concept from which the level of importance of requirements defined by users could be validated. These impacts have led to the proposal of a new vision in the modeling of business processes. This vision was named triangular model of business processes. However, we did not place emphasis on indicators related to business objects. This is already the subject of ongoing work. In the coming days, we plan:

-To propose an extension of business components, so that they support the concepts of satisfaction and performance indicators;

-To Define passage rules from a business process requirement model to business component model.

-To define a platform for identifying a system requirements model and in the same vein, identify reusable requirements; enhance the work on selection of software components.

The purpose of all these works is to implement a componentbased development platform from a requirements specification closer to the human language and which takes into account the expectations of the consumers of the services rendered by the business process. This should surely permit us minimize misunderstandings between developers and business executives, and produce systems based on software components of lower costs while mastering the changing requirement in a business process. 


\section{REFERENCES}

[1] R. Atsa Etoundi, M. Fouda Ndjodo, Christian Lopez Atouba, "A Goal Oriented Approach or the Definition of a Business Process Requirement Model", International Journal of Computer Applications 9(7):1-7, 2010

[2] R. Atsa Etoundi, M. Fouda Ndjodo, Christian Lopez Atouba, "A Goal Based Approach for QFD Refinement in Systematizing and Identifying Business Process Requirements", IJCSI, 2010

[3] Mouhamed Diouf, «Spécification Et Mise En œuvre D'un Formalisme De Règles Métier», thèse ${ }^{\circ} 3507$, Université Bordeaux I, décembre 2007.

[4] Hang-Wai Law, Meng Hua, "Using Quality Fonction Deployment in Singulation Process Analysis", Engineering Letters, February 2007.

[5] Anis Ferchichi, « contribution a l'intégration des processus métiers : application a la mise en place d'un référentiel qualité multi-vues», thèse, Université Bordeaux I, 2008.

[6] Lubars, M., Potts, C., Richer, C.: A review of the state of the practice in requirements modeling.Proc. IEEE Symp. Requirements Engineering, San Diego 1993.

[7] Karen Mc Graw, Karan Harbison, User Centered Requirements, The Scenario-Based Engineering Process. Lawrence Erlbaum Associates Publishers, 1997.

[8] The Standish Group, Chaos. Standish Group Internal Report, http://www.standishgroup.com/chaos.html, 1995

[9] R. Atsa Etoundi, M. Fouda Ndjodo, «Human Resource Constraints driven Virtual Workflow Specification », IEEE SITIS pp 176-182, 2005.

[10] R. Atsa Etoundi, M. Fouda Ndjodo, «Feature-Oriented Business Process and Workflow », IEEE SITIS pp 114121, 2005.

[11] Farida Semmak, Joël Brunet, "Un métamodèle orienté buts pour spécifier les besoins d'un domaine», 23e Congrès INFORSID, pp 115-132, mai 2005.

[12] Lubars, M., Potts, C., Richer, C.: A review of the state of the practice in requirements modeling.Proc. IEEE Symp. Requirements Engineering, San Diego 1993.

[13] Karen Mc Graw, Karan Harbison, User Centered Requirements, The Scenario-Based Engineering Process. Lawrence Erlbaum Associates Publishers, 1997.

[14] Farida Semmak, Joël Brunet, «Un métamodèle orienté buts pour spécifier les besoins d'un domaine», 23e Congrès INFORSID, pp 115-132, mai 2005.

[15] Cardozo R., An Experimental Study of Customer Effort, Expectation and Satisfaction, Journal of Marketing Research, 2, pp: 244-249, 1965.

[16] Gronroos Christian, Strategic Management and Marketing in the Service Sector, Helsingfors: Swedish School of Economics and Racine.- Administration, 1982

[17] Grönroos C. A Service Quality Model and its Marketing Implications, European Journal of Marketing, 4, pp: 3644, 1984.
[18] Parasuraman A., Zeithaml Valarie A. and Berry Leonard L., SERVQUAL : A Multiple-Item Scale for Measuring Consumer Perceptions of Service Quality, Journal of Retailing, 64, 1, pp: 12-40, 1988.

[19] Parasuraman A., Berry Leonard L. and Zeithaml Valarie A., A Conceptual Model of Service Quality and its Implications for Future Research, Journal of Marketing, 49, pp: 41-50, 1985.

[20] Oliver Richard L. (1980), A Cognitive Model of the Antecedents and Consequences of Satisfaction Decisions, Journal of Marketing Research, 17, pp: 460-469,1980.

[21] Parasuraman A., Berry Leonard L. and Zeithaml Valarie A., Refinementand Reassessment of the SERVQUAL Scale, Journal of Retailing, 67, 4, pp: 420-450, 1991.

[22] Howard et Sheth (1969), The Theory of Buyer Behavior, New York, John Wiley and sons.

[23] Eiglier P. et Langeard E., Servuction - Le marketing des services, Stratégie et Management, Paris, Mc Graw-Hill, 1987.

[24] Olshavsky R.W. et Miller J.A. (1972), Consumer Expectations, Product Performance and Perceived Product Quality, Journal of Marketing Research, 9, pp: 19-21, 1987.

[25] Churchill G.A. et Surprenant C., An Investigation into the Determinants of Customer Satisfaction, Journal of Marketing Research, 19, pp : 491-504, 1982.

[26] Hafedh Mili, Guitta Bou Jaoude, Eric lefebvre, Guy Tremblay, and Alex Petrenko. Business process modeling languages : Sorting through the alphabet soup. Rapport de recherché, Département d'Informatique, UQAM, Janvier 2004.

[27] Bernard Debauche and Patrick Megard. BPM Business Process Management : Pilotage métier de l'entreprise Hermes Science Publications, 2004.

[28] M. Fouda Ndjodo, P. Essawe Ndedi, R. Atsa Etoundi, An Interperspective-Oriented Business Process Modeling Approach, Bider and al. (Eds): BPMDS 2010 and EMMSAD 2010, LNBIP 50, pp. 145-156, 2010 , Springer-Verlag Berlin Heidelberg 2010.

[29] Carman James M., Consumer Perceptions of Service Quality: An Assessment of the SERVQUAL Dimensions, Journal of Retailing, 66, printemps, pp : 3355,1990

[30] Alain Fernandez, Les nouveaux tableaux de bord des managers: Le projet décisionnel dans sa totalité, Quatrième édition, Groupe Eyrolles, ISBN : 978-2-21254124-3, 2008

[31] R. Atsa Etoundi, M. Fouda Ndjodo, Christian Lopez Atouba, "A Model Based Business Process Requirement Rule Specification, International Journal of Computer Applications 11(9):17-24, December 2010.

[32] Atsa Etoundi Roger, Fouda Ndjodo Marcel, Atouba Christian Lopez, Business Process Requirement Engineering. International Journal on Computer Science and Engineering, volume 2, $\mathrm{n}^{\circ}$ 9, December 2010. 